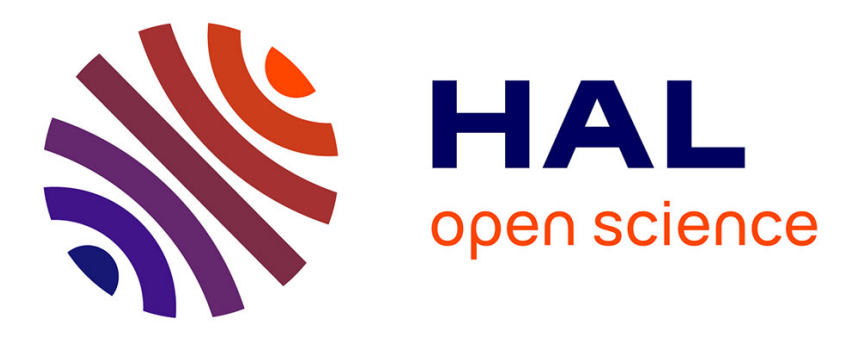

\title{
Physiologically Based Modeling of Food Digestion and Intestinal Microbiota: State of the Art and Future Challenges. An INFOGEST Review
}

\author{
Steven Le Feunteun, Ahmed Al-Razaz, Matthijs Dekker, Erwin George, \\ Béatrice Laroche, George van Aken
}

\section{To cite this version:}

Steven Le Feunteun, Ahmed Al-Razaz, Matthijs Dekker, Erwin George, Béatrice Laroche, et al.. Physiologically Based Modeling of Food Digestion and Intestinal Microbiota: State of the Art and Future Challenges. An INFOGEST Review. Annual Review of Food Science and Technology, 2021, 12, pp.16/1-16/19. 10.1146/annurev-food-070620- . hal-03117715

\author{
HAL Id: hal-03117715 \\ https://hal.inrae.fr/hal-03117715
}

Submitted on 21 Jan 2021

HAL is a multi-disciplinary open access archive for the deposit and dissemination of scientific research documents, whether they are published or not. The documents may come from teaching and research institutions in France or abroad, or from public or private research centers.
L'archive ouverte pluridisciplinaire HAL, est destinée au dépôt et à la diffusion de documents scientifiques de niveau recherche, publiés ou non, émanant des établissements d'enseignement et de recherche français ou étrangers, des laboratoires publics ou privés.

\section{(1) (1) $\$$}

Distributed under a Creative Commons Attribution - NonCommercial - NoDerivatives 44.0 


\title{
AR REVIEWS
}

\section{Annual Review of Food Science and Tecbnology Physiologically Based Modeling of Food Digestion and Intestinal Microbiota: State of the Art and Future Challenges. An INFOGEST Review}

\author{
Steven Le Feunteun, ${ }^{1}$ Ahmed Al-Razaz, ${ }^{2}$ \\ Matthijs Dekker, ${ }^{3}$ Erwin George, ${ }^{4}$ \\ Beatrice Laroche, ${ }^{5}$ and George van Aken ${ }^{6}$ \\ ${ }^{1}$ STLO, INRAE, Institut Agro, 35042 Rennes, France; email: steven.le-feunteun@inrae.fr \\ ${ }^{2}$ Essex Pathways, University of Essex, CO4 3SQ Colchester, United Kingdom; \\ email: ahmed.alrazaz@essex.ac.uk \\ ${ }^{3}$ Food Quality and Design Group, Department of Agrotechnology and Food Sciences, \\ Wageningen University, 6700 AA Wageningen, The Netherlands; email: matthijs.dekker@wur.nl \\ ${ }^{4}$ School of Computing and Mathematical Sciences, University of Greenwich, SE10 9LS \\ London, United Kingdom; email: e.george@greenwich.ac.uk \\ ${ }^{5}$ Université Paris-Saclay, INRAE, MaIAGE, 78350 Jouy-en-Josas, France; \\ email: beatrice.laroche@inrae.f \\ ${ }^{6}$ Cosun Innovation Center, Royal Cosun, 4670 VA Dinteloord, The Netherlands; \\ email: george.van.aken@cosun.com
}

Annu. Rev. Food Sci. Technol. 2021. 12:16.1-16.19

The Annual Review of Food Science and Technology is online at food.annualreviews.org

https://doi.org/10.1146/annurev-food-070620124140

Copyright (? 2021 by Annual Reviews. All rights reserved

\section{Keywords}

in silico, gastrointestinal tract, digestion, transit, absorption, microbiota

\section{Abstract}

This review focuses on modeling methodologies of the gastrointestinal tract during digestion that have adopted a systems-view approach and, more particularly, on physiologically based compartmental models of food digestion and host-diet-microbiota interactions. This type of modeling appears very promising for integrating the complex stream of mechanisms that must be considered and retrieving a full picture of the digestion process from mouth to colon. We may expect these approaches to become more and more accurate in the future and to serve as a useful means of understanding the physicochemical processes occurring in the gastrointestinal 
tract, interpreting postprandial in vivo data, making relevant predictions, and designing healthier foods. This review intends to provide a scientific and historical background of this field of research, before discussing the future challenges and potential benefits of the establishment of such a model to study and predict food digestion and absorption in humans.

\section{INTRODUCTION}

Investigating the effects of food composition and structure on the fate of food in the human gastrointestinal (GI) tract is necessary for food developers and nutritionists to measure and optimize nutrient availability. Although the final assessments needed to make health, nutritional, and functional claims must always involve human studies, these are very expensive, time consuming, and constrained by medical and ethical assessments of their acceptance. As alternatives to human and animal studies, in vitro experiments, which include both static and dynamic digestion models, have become very popular in recent years. The international INFOGEST network has been particularly active in this field, notably regarding the harmonization and validation of in vitro digestion protocols (Brodkorb et al. 2019, Minekus et al. 2014, Mulet-Cabero et al. 2020) and the correlation of the results obtained with these protocols to in vivo data (Bohn et al. 2018, Dupont et al. 2019, Egger et al. 2016). Although in vitro studies have demonstrated success in evaluating the effects of food properties on digestion, they miss certain properties related to the way the body dynamically controls the digestion process. Computer modeling of the digestion process appears a very promising means to expand our understanding of the relationships between foods (or diets) and the overall function of the human GI tract and post-absorptive processes.

The present review focuses on mathematical models of the GI tract that have adopted a systems-based approach, and, more particularly, on physiologically based compartmental modeling. These methods have been applied in the veterinarian area related to animal feed sciences (Bannink et al. 2006, Bastianelli et al. 1996, Rivest et al. 2000, Strathe et al. 2008, Usry et al. 1991) and are widely used in pharmaceutics for the development of orally administered pharmaceuticals (Chetty et al. 2018, Yu 1999, Zhuang \& Lu 2016). Following these works, compartmental models related to food digestion and/or postprandial metabolism have also been proposed. This review aims to provide a state-of-the-art overview of this area, before discussing future challenges associated with the modeling of food digestion and its metabolic repercussions in humans.

Section 2 highlights the compartmental organization of current pharmacokinetic models and their contribution to pharmaceutical development. Section 3 describes features that have been added to this compartmental approach for the modeling of food digestion and absorption. Compared to the existing pharmacokinetic models, much more attention is needed to introduce the complexity of food materials consisting of a multiscale structure containing many components. Section 4 discusses the overall progress made in food digestion compartmental modeling and provides an opinion on what future developments are needed.

\section{MODELING THE DIGESTIVE TRACT}

Computer simulation and mathematical modeling are common techniques that have proven their worth in many disciplines. Generally speaking, a mathematical model aims to describe aspects of a real-life phenomenon by using fundamental assumptions to purposefully remove unnecessary details. Consequently, a mathematical model is merely an analogy or caricature of the phenomenon and as such is not completely accurate in its description of the real-life situation. However, models can still accurately depict some important system behaviors and hence are very useful. 
In the current discussion, we focus on dynamic (as opposed to static) models in which timedependent changes are accounted for and that are typically characterized by systems of differential equations. Another key categorization of mathematical models is on a scale from empirical to mechanistic. Purely empirical models make no assumptions about the mechanisms controlling the system behavior but focus solely on replicating the data. As such, they commonly fail when used for predictions outside the range of data used to establish them. Examples of these kinds of pharmacokinetic models include those with sum-of-inverse-Gaussian functions to describe drug absorption profiles (Csajka et al. 2005) or Bayesian p-splines to estimate pharmacokinetic parameters (Jullion et al. 2009). By contrast, a purely mechanistic model incorporates physiologically based assumptions about the mechanisms controlling the system. For example, each compartment has some specific physiological identification, as do the parameters associated with each compartment. Mechanistic models are predominantly applied to data to investigate whether the model mirrors the experimentally observed trends. Model parameters are estimated with caution to preserve the scientific soundness of the model. One advantage of a mechanistic model over an empirical model is its ability to extrapolate beyond the data with which it was built. In pharmacokinetics, many models are semimechanistic, often having physiologically relevant compartments but at least some of the parameters are determined empirically.

The research on compartmental modeling has led to several commercial packages for pharmacological modeling of the delivery of orally administrated drugs, as has been reviewed by others (Chetty et al. 2018, Li et al. 2018, Pentafragka et al. 2019). The difficulty in these physiologically based pharmacokinetic (PBPK) models is that evaluation of model parameters is usually empirical rather than based on the underlying mechanisms (Aarons 2005). Nonetheless, such mathematical methodologies have been successfully developed in the pharmaceutical industry to the point that they are now often used in advance of clinical trials to help optimize the trial design for a given objective (Danhof et al. 2008, van der Graaf \& Benson 2011).

Similar developments have been proposed to model food digestion and absorption in the alimentary tract with two main approaches: continuum and compartmental models. Continuum models take the tubular organization of the GI tract as a starting point and mathematically describe the functional variation, transit, digestion, and absorption by propulsive contractions as functions of the location (Labarthe et al. 2019; Moxon et al. 2016, 2017; Taghipoor et al. 2012, 2014). These models can simultaneously consider different aspects of digestion, such as the transit of the food along the intestine, enzymatic degradation of foods, and nutrient absorption. However, these models involve systems of partial differential equations structured in space, which increase in complexity for each digestive feature added, requiring efficient numeric solvers. The remainder of this review is restricted to compartmental modeling because this is the most widely used in pharmacokinetic modeling (Li et al. 2018, Pentafragka et al. 2019). In these models, the GI tract is represented by a series of compartments with different functionalities and digestive conditions ( $\mathrm{pH}$, enzyme concentrations, etc.), considering, for instance, the mouth, the stomach, the small intestine, and/or the large intestine. They sometimes include more gradual variations, e.g., the duodenum, jejunum, and ileum of the small intestine.

\section{FOOD DIGESTION MODELS}

\subsection{What Phenomena Should Be Modeled?}

Although compartmental models have found wide applications in pharmacokinetics, they are not directly suited for application in food digestion research. They usually assume specific generalized conditions in either the fasted or fed states but lack a suitable description of the meal properties. Therefore, a step in advancing these models toward application in food digestion studies is to 
Table 1 Overview of the phenomena and the important food properties that should be considered when developing a multicompartment model of food digestion and absorption

\begin{tabular}{|c|c|c|}
\hline $\begin{array}{l}\text { Physiological } \\
\text { compartment }\end{array}$ & Main phenomena to be modeled & Important food properties \\
\hline Oral & $\begin{array}{l}\text { Mechanical breakdown } \\
\text { Saliva incorporation (amylase, water, mucus) } \\
\text { Enzymatic hydrolysis of starch }\end{array}$ & $\begin{array}{l}\text { Mechanical and rheological properties } \\
\text { Structure (mostly macro) } \\
\text { Composition (dry content, } \mathrm{pH}, \text { nutrients) }\end{array}$ \\
\hline Gastric & $\begin{array}{l}\text { Gastric secretion (enzymes, } \mathrm{HCl}, \text { mucus) } \\
\text { pH drop } \\
\text { Enzymatic hydrolysis of starch, protein, and lipid } \\
\text { Mechanical breakdown } \\
\text { Mixing and sieving } \\
\text { Phase separation (sedimentation, creaming) } \\
\text { Controlled flow into duodenum }\end{array}$ & $\begin{array}{l}\text { Mechanical and rheological properties } \\
\text { Structure (all scales: particle size and } \\
\text { density, emulsion stability, } \\
\text { microstructures, state of nutrients, etc.) } \\
\text { Composition } \\
\text { Buffering capacity } \\
\text { Intermolecular interactions }\end{array}$ \\
\hline Duodenum & $\begin{array}{l}\text { Multiple secretions (pancreatic enzymes, bile, } \\
\quad \text { bicarbonate, water, mucus) } \\
\mathrm{pH} \text { increase } \\
\text { Enzymatic hydrolysis of starch, protein, and lipid } \\
\text { Absorption of water and nutrients } \\
\text { Mixing } \\
\text { Residence time }\end{array}$ & $\begin{array}{l}\text { Structure (all scales) } \\
\text { Composition } \\
\text { Buffering capacity } \\
\text { Intermolecular interactions }\end{array}$ \\
\hline Jejunum & $\begin{array}{l}\text { Intestinal secretion (water, bicarbonate, mucus) } \\
\text { Enzymatic hydrolysis of starch, protein, and lipid } \\
\text { Absorption of water and nutrients } \\
\text { Mixing } \\
\text { Residence time }\end{array}$ & $\begin{array}{l}\text { Structure (all scales) } \\
\text { Composition } \\
\text { Intermolecular interactions }\end{array}$ \\
\hline Ileum & $\begin{array}{l}\text { Intestinal secretion (water, bicarbonate, mucus) } \\
\text { Enzymatic hydrolysis of starch, protein, and lipid } \\
\text { Absorption of water, nutrients and bile salts } \\
\text { Mixing } \\
\text { Residence time }\end{array}$ & $\begin{array}{l}\text { Structure (all scales) } \\
\text { Composition } \\
\text { Intermolecular interactions }\end{array}$ \\
\hline $\begin{array}{l}\text { Ascending, transverse, } \\
\text { and descending } \\
\text { colon }\end{array}$ & $\begin{array}{l}\text { Intestinal secretion (water, bicarbonate, mucus) } \\
\text { Microbial growth and metabolism and the way they are } \\
\text { affected by material entering the colon } \\
\text { Absorption of water and microbial metabolites such as } \\
\text { short-chain fatty acids } \\
\text { Mixing } \\
\text { Residence time }\end{array}$ & $\begin{array}{l}\text { Composition (fiber, polyphenols, polyols, } \\
\text { etc.) } \\
\text { Interaction of components with the } \\
\text { microbiota }\end{array}$ \\
\hline
\end{tabular}

include the effects of food composition (size, caloric density, buffering effects, etc.) and structure (liquid or solid, inner substructures, etc.) on the modeled mechanisms. These mechanisms are outlined in Table 1 and have recently led to a public shared reference library focusing on related in silico models (INFOGEST In Silico Work. Group 2019). Examples of how these phenomena are becoming integrated into mathematical food digestion models are provided below. Beyond these general considerations, an appropriate level of detail is needed to simulate the fate of nutrients from the foods to peripheral blood. As schematically represented in Figure $\mathbf{1}$ for starch digestion (omitting proteins and lipids to keep the figure simple), we believe that at least the lumen and the gut wall should be distinguished and that the original substrates and end products of the enzymatic reactions should be considered. A further possible step, not considered in Table $\mathbf{1}$ and Figure 1, 

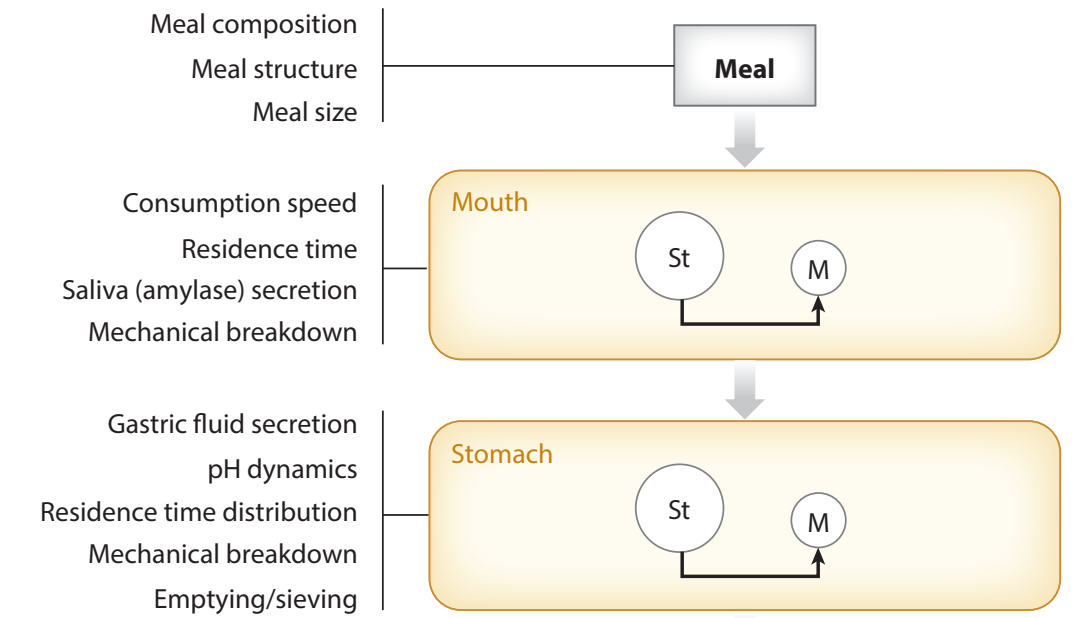

Emptying/sieving

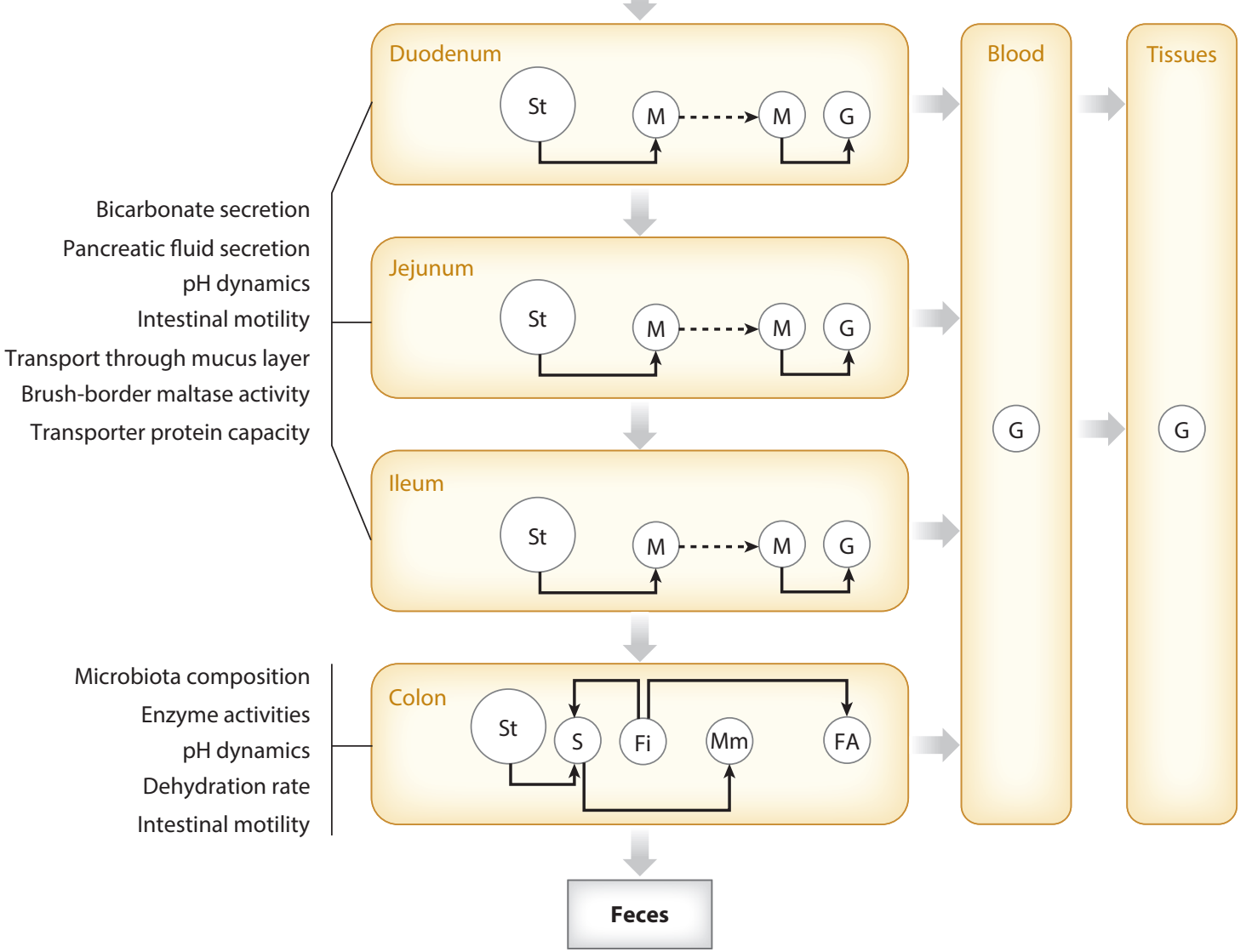

Figure 1

Schematic representation of starch digestion and important processes to be included in a multicompartment model (omitting proteins and lipids to keep the figure simple). Solid arrows indicate transformations, and dashed arrows indicate transport from lumen to the brush border through the mucus layer. Abbreviations: FA, short-chain fatty acids; Fi, fiber; G, glucose; M, end products of amylolytic reactions (maltose, maltotriose, and $\alpha$-limit dextrins); $M$ m, microbial mass; $\mathrm{S}$, various sugars; $\mathrm{St}$, starch. 
consists of integrating the physiology that controls the GI conditions of digestion (e.g., feedback mechanisms). This currently relatively unexplored aspect is discussed in Section 4.2.

\subsection{Integration of the Food Composition in Nutrients}

Several compartmental models have been developed in relation to food digestion in humans and feed digestion in animals. Examples range from models addressing the flow and mass transfer of digesta along the GI tract (Moxon et al. 2016, Rivest et al. 2000, Taghipoor et al. 2012, Usry et al. 1991) to those addressing the metabolic fate of absorbed nutrients in the host (Fouillet et al. 2009, Mc Auley \& Mooney 2015). However, these models do not distinguish the different food components, as they usually focus on one kind of nutrient only. To simulate the digestion of complex foods or meals, the first necessary step is to integrate their nutritional composition.

The first attempts to integrate all the main constituents of food products in a compartmental model of digestion arose from animal feed science. A good example thereof is the modeling of digestion and absorption in pigs by Bastianelli et al. (1996), which was extended and refined by Strathe et al. (2008). In the version proposed by Strathe et al. (2008), a total of 38 coupled compartments are considered, consisting of four main GI segments (stomach, proximal and distal small intestine, and large intestine) subdivided into the major biochemical compounds (e.g., endogenous and dietary proteins, amino acids, starch and sugars, dietary lipids and fatty acids, degradable and undegradable dietary fiber). In this model, gastric emptying and intestinal transit are represented by mass action, and the rates of enzymatic degradation and absorption of nutrients are described by saturation kinetics. Model parameterization was performed based on a literature collection on the digestive physiology of pigs. Its stability was evaluated by sensitivity analysis (SA) (see Section 3.5), and the quality of its predictions was assessed by comparisons with published results. Overall, the model appeared quite robust to variations of the studied parameters and provided reasonably good predictions of the digestibility of the main food components (except lipids) as well as the absorption profile of the studied nutrients (glucose, amino acids, and volatile fatty acids).

To the best of our knowledge, no similar model (i.e., with all nutrients) has yet been published for food or meal digestion and absorption in humans, although we are aware of at least one ongoing development (van Aken 2020), which is exemplified in Section 4.4. Human physiologically based compartment models that include GI-tract compartments have been, nonetheless, proposed to predict the metabolic fate of each of the main nutrients independently: proteins, lipids, and carbohydrates (see, e.g., Fouillet et al. 2009, Jelic et al. 2009, Rozendaal et al. 2018). Although these models do not primarily focus on the mechanisms that transform food into absorbable nutrients, they are important extensions of the previously described approaches. A better representation of the functioning of the GI tract during digestion, using compartmentalization suitable to all kinds of nutrients, would thus directly serve postprandial metabolic models via integration with other physiological compartments, such as splanchnic and peripheral organs. As an example, a total of 13 anatomical compartments, 9 of which represent non-GI-tract compartments, were used by Fouillet et al. (2009) to simulate the dynamics of meal nitrogen absorption, splanchnic uptake, and metabolism, with subsequent peripheral transfer and deposition. Because the number of compartments and associated parameters increases remarkably in such models, error compensations are likely to occur and remain undetected. Beyond computational issues, this is another important reason why building and validating a whole-body and all-nutrient physiologically based model remain a difficult challenge.

\subsection{Integration of Food Structure Effects}

The amount and the dynamics of nutrient uptake after a meal are also largely governed by food structure effects (Bornhorst et al. 2016, Dupont et al. 2018). Meals differing only in their 
processing conditions can even affect intestinal mucosa parameters and the microbiota composition in the colon (Beaumont et al. 2017, Oberli et al. 2018). These findings result from the influence of food structure on key mechanisms taking place in the upper parts of the GI tract: food comminution, food mixing with digestive fluids, gastric-emptying kinetics, etc. For instance, it is well known that the gastric-emptying kinetics of liquid meals follow an exponential behavior, whereas a lag phase is classically observed with solid meals. To properly simulate the breakdown of solid foods and their mixing with digestive fluids, computational solid mechanics and hydrodynamic modeling of oral and gastric processing can be employed (Ferrua \& Singh 2010, 2015; Harrison et al. 2014, 2018). However, current oral and gastric computational models do not account yet for secretions and enzymatic reaction(s) and have focused only on the gastric behavior of liquids, with no or few discrete solid particles. Moreover, these are computationally expensive and are not easily compatible with systems modeling approaches. This is why other means are classically used to try to reproduce food structural effects in compartmental models.

The most commonly employed strategy is to simulate gastric-emptying kinetics with empirical equations. The oldest one, which is still among the most commonly employed, is the Elashoff equation (Elashoff et al. 1982), which assumes that the fraction of the meal in the stomach follows a power exponential decay. As recently reviewed by Muttakin et al. (2019), other empirical equations have been proposed to simulate the biphasic nature of gastric emptying that is generally observed with solid foods (Siegel et al. 1988, Kong \& Singh 2009).

Another strategy assumes several gastric subcompartments (Dalla Man et al. 2006, Le Feunteun et al. 2014, Sicard et al. 2018, van Bentum \& Nelson 2011) to incorporate nonideal mixing. For instance, two gastric subcompartments were used by Le Feunteun et al. (2014) to simulate protein digestion of differently structured dairy protein matrices in mini-pigs: one to represent the matter that is retained within the stomach (large particles, matter too far from the pylorus to pass through, etc.), and another to represent the matter that is well mixed with the secretions and is ready to be emptied. Such a strategy enables representing heterogeneous gastric content with a delayed emptying for solid foods while avoiding simulating unreasonable gastric volumes or secretions.

However, none of these above options is perfect. New developments thus remain to be proposed to relate food structural properties more directly with their behavior in the upper parts of the GI tract and, in particular, with the kinetics of gastric emptying and enzymatic degradation. Nonetheless, the growing interest of food scientists in the fate of food during digestion is currently leading to an increasing body of physical models on the behavior of foods under the dynamic conditions of the GI tract. Examples include models of the buffering capacity of foods in the stomach (Luo et al. 2018, Mennah-Govela et al. 2020, Mennah-Govela et al. 2019), the swelling of protein gels (van der Sman et al. 2020), the softening of carbohydrate foods (Drechsler \& Bornhorst 2018), the mechanical breakdown of solid foods (Drechsler \& Ferrua 2016), and mass transfer and absorption in the intestine (Moxon et al. 2016, Taghipoor et al. 2012, 2014). A reaction-diffusion model of gastric digestion of meat proteins, which takes into account enzyme and proton diffusion into bolus particles with consideration of buffering effects, was also recently proposed by Sicard et al. (2018). Given that mechanistic digestion models that aim to integrate the physical properties of foods are still in their early stages and that this research area has recently become very active, it is expected that important progress will soon be made on the modeling of food structure effects on digestion.

\subsection{Integration of the Microbiota}

The lower part of the digestive system hosts the intestinal microbiota. This complex microbial community not only processes nondigestible dietary residues by anaerobic digestion reactions 
in the colon (Korpela 2018) but also maintains a complex dialog with the host and plays a very important protective role against pathogenic microorganisms (Guarner \& Malagelada 2003).

Some large-scale (whole digestive system or body) digestion models integrating the ecological and metabolic dynamics of microbes have been developed to provide a detailed representation of host-diet-microbe interplays. These models are mainly based on two reductive representations of the intestinal microbiota. The first one focuses on functional traits of microorganisms and models the microbiota by a small number of functional populations, assuming high functional redundancy in the ecosystem. The second focuses on a few dominant species, whose metabolism is modeled in detail.

A prototypal example of the first category is the model of fiber digestion in the large intestine developed by Muñoz-Tamayo et al. (2010). The colon is divided into compartments corresponding to physiological regions in which a simplified biochemical reactions network models fiber degradation and short-chain fatty acid (SCFA) production. The local microbiota is structured into functional populations that catalyze these reactions. The model includes transport, diffusion, and absorption between compartments or between the colon and the host. Its parameters were drawn from the literature and from in vitro culture experiments. This work aimed to synthesize available knowledge in a single model and qualitatively reproduce simple nutritional scenarios such as variation of dietary fiber level and its influence on SCFA production and colon transit. On the basis of the same concept of functional microbial populations, several other models were proposed, e.g., a model for the proximal colon in humans (Motelica-Wagenaar et al. 2014); an in vitro model rumen focused on $\mathrm{pH}$, hydrogen metabolism, and hexose and amino acids utilization (MuñozTamayo et al. 2016); and continuous, spatially explicit models coupling microbial metabolism, fiber digestion, and fluid dynamics in the colon (Cremer et al. 2017, Labarthe et al. 2019). Kettle and coworkers (Kettle et al. 2015,2018) developed an integrated model for the 10 major bacterial functional groups in the human colon. The growth kinetics and major metabolism of these groups were modeled by taking into account their individual substrate specificities, metabolic pathways, and $\mathrm{pH}$ effects. This model accounts for individual differences in terms of microbiota composition and consists of a large system of differential equations on microbial growth, substrates, and metabolites concentrations. Recently, a multicompartment modeling tool was developed in a widely used programming language (R) called microPop (Kettle et al. 2018) to simulate microbiota kinetics in different compartments of the human colon.

Models in the second category (i.e., those with few dominant species, whose metabolism is modeled in detail) result from the rapid and successful development of constraint-based genomescale metabolic models (GEMs) of microorganisms (Kim et al. 2017, Thiele \& Palsson 2010). In these models, the internal metabolic flux partition of each cell is assumed to be at a quasi-steady state and must be computed as soon as the external conditions and uptake rates change. There are several methods to do this. One of the most popular is flux balance analysis (Orth et al. 2010), for which highly efficient tools such as the MATLAB Toolbox COBRA (Heirendt et al. 2019) can be used. GEM-based microbiota models are recent; one example is the model proposed by Heinken et al. (2013), which includes 15 prevalent genomes and the host epithelial cells. This model computes the metabolic partition between the different cells given input fluxes of nutrients and can be coupled with compartment models.

Advances in culture-free sequencing techniques in the past 20 years, such as 16 SrDNA amplicon sequencing or whole-genome shotgun (WGS) sequencing allows access to the taxonomic and functional profiling of large intestine microbiota for human (Li et al. 2014), pig (Xiao et al. 2016), or mouse (Xiao et al. 2015). For both types of models mentioned above, recent efforts have been proposed to integrate the enormous taxonomic and functional diversity in the digestive microbiota, together with the wide variety of indigested dietary fibers (Korpela 2018). A first attempt to 
link functional population with WGS metagenomics can be found in Raguideau et al. (2016), and a complete MATLAB toolbox to build and simulate GEM models of host and microbiota based on $16 \mathrm{~S}$ data has recently been released by Baldini et al. (2019).

\subsection{Mathematical Tools for Predictive Modeling}

Predictive compartmental models are characterized by their ability to cope with uncertainties related to environmental variability (e.g., formulation, structure, and preparation of drugs or foods) and interindividual variability (e.g., age, weight, sex, and physical condition of the subject) and finally with the more or less accurate knowledge and representation of the phenomena involved in the digestion and absorption of nutrients. Indeed, the ultimate goal of predictive modeling is to provide prediction intervals in which quantities of interest are located when simulating an uptake scenario, with associated probability and modulation by the characteristics of foods and individuals. Models for food digestion and absorption are often large-sized, complex models with limited observations, especially in vivo. Moreover, the available information is heterogeneous, in that it may be obtained via very different measurement techniques and come from different experiments or subjects. This is why building predictive models is a major issue in the field and calls for the use of sophisticated mathematical tools. For instance, parameter estimation, in which descriptors, such as mean, variance, and covariance, of the statistical distribution of the model parameter are estimated, is a crucial step. Many generic or specialized software or programs are available, implementing methods ranging from nonlinear least-squares regression to Bayesian estimation (Balsa-Canto et al. 2016, Haario et al. 2006, Raue et al. 2015). Other aspects, such as interindividual variability, must also be accounted for when dealing with population studies. This can be done through mixed-effect models (Lavielle 2014), which can incorporate covariates.

In establishing a comprehensive food digestion model, we may also insist on the usefulness of conducting sensitivity analysis (SA), which is a highly relevant tool for models in which several phenomena may interact in a complex way to produce an overall behavior. SA enables highlighting parameters whose variation has a significant influence on the model responses. Hence, parameters distinguished as significant can then be estimated experimentally and/or clinically whenever possible, whereas the remaining must be taken from the literature or approximated. SA may also lead to the total elimination of some model parameters considered insignificant (Manca 2018).

Methods employed for SA can be either local or global. Local methods estimate the effect of small parameter variations on a model response and are primarily used in steady-state models. Global SA (GSA) (Saltelli et al. 2008, Sudret 2008), by contrast, is able to examine the entire parameter space, manage nonlinearities well, and provide a complete ranking of parameters by significance. GSA is a numerical exploration approach based on statistical theory that aims to understand the influence of parameters on selected model outputs, for which several software tools are available (see, e.g., Iooss et al. 2019, Saturnino et al. 2019). For example, an application of SA can be found in Labarthe et al. (2019), where GSA was performed with a model of the spatial repartition of the bacterial population in the human colon and helped in understanding the influence and relative strength of different factors, including peristalsis, fiber intake, and mucus viscosity, on the total bacterial abundance along the colon.

In GSA, the parameter space is sampled in an appropriate way and the corresponding model outputs are subsequently analyzed with various methods, the most popular of which are the calculation of Sobol indices and Partial Rank Correlation Coefficients (PRCC) [see Iooss \& Lemaitre (2015) for a complete review]. For complex models, low-cost computational screening methods can be used to eliminate insignificant variables prior to employing GSA for the remaining parameters, such as the screening method of Morris (1991). 


\section{FUTURE CHALLENGES}

\subsection{Toward a More Complete Model of Food Transit and Absorption}

As depicted in Section 3, mathematical models related to various aspects of the GI tract have been proposed from different scientific fields (pharmaceutics, nutrition, animal sciences, food sciences, microbiology). Several pieces are thus available to start building a systems model of the functioning of the human GI tract during digestion. Its skeleton could be inspired by the works already undertaken in pharmaceutics and animal sciences before integrating the most relevant and promising modeling efforts.

For instance, the model proposed by Strathe et al. (2008) for pigs already accounts for all the main nutrients of a meal, with consideration of their transit, hydrolysis, absorption kinetics, nutrient degradation, and some aspects of the microbial metabolism in the large intestine. We may, therefore, assume that a comparable model organization, with some adaptations related to human physiology, could constitute a fair starting point before refining and completing the model structure and its underlying hypotheses. It has also been shown that gastric-emptying kinetics can be predicted by assuming a nutrient feedback mechanism in the proximal small intestine (Hunt \& Stubbs 1975, Moxon et al. 2017), leading to a mean rate of caloric emptying of approximately $2.5 \mathrm{kcal} / \mathrm{min}$ in humans (Hunt et al. 1985). This strategy seems much more elegant than empirical equations or mass action laws to predict gastric emptying, as recently proposed in a standardized semi-dynamic protocol for in vitro digestion (Mulet-Cabero et al. 2020). However, to the best of the authors' knowledge, this modeling strategy has not yet been applied in published compartmental models of food digestion and absorption. Recent developments in the modeling of enzymatic hydrolysis are also providing new means to take into account some key properties of the main nutrients, with nonempirical relationships between the model parameters and the food properties. These models mostly rely on the consideration that different rates of hydrolysis can be assumed for different subclasses of the considered nutrient (i.e., more or less resistant and/or accessible fractions). Examples have been proposed for lipids (Giang et al. 2016), proteins (Barros \& Xavier Malcata 2004, Kondjoyan et al. 2015), and starch (Edwards et al. 2014, Li et al. 2019, Meraz et al. 2019), with promising results. These approaches are interesting ways to incorporate compositional (e.g., the fatty acid composition of lipids) and structural (e.g., percentage of gelatinized starch) considerations of the studied food(s) into enzymatic hydrolysis models. A relevant model of food digestion in the upper parts of the GI tract would also enable better predictions of the unabsorbed meal fraction and hence be valuable for the modeling of colon microbiota functioning.

The present review also highlights the current lack of modeling tools and gaps in knowledge. In particular, new developments would be very welcome to relate the oral and gastric behavior of solid foods more directly with their known or measured properties. We may nonetheless be confident that the growing interest of food scientists and modelers in this research area will rapidly bear fruit, without forgetting that empirical relationships can be of use while awaiting mechanistic representations. A great many pieces are thus already available to start building a framework to test and improve our understanding of food digestion in humans.

Here, we highlight the ongoing development of a multicompartment model of digestion in humans (van Aken 2020) that considers all nutrients, most of the phenomena listed in Table 1, and even some physiological feedback mechanisms (see Section 4.2). As an illustrative example of the capabilities of such a model, Figure 2 compares its predictions with the human data obtained by Eelderink et al. (2012) on blood glucose and insulin excursions after a bread meal and a pasta meal. As observed experimentally, the model could reproduce a slightly lower glycemic peak and a much reduced insulinemic peak for the pasta meal compared to the bread meal. In the 

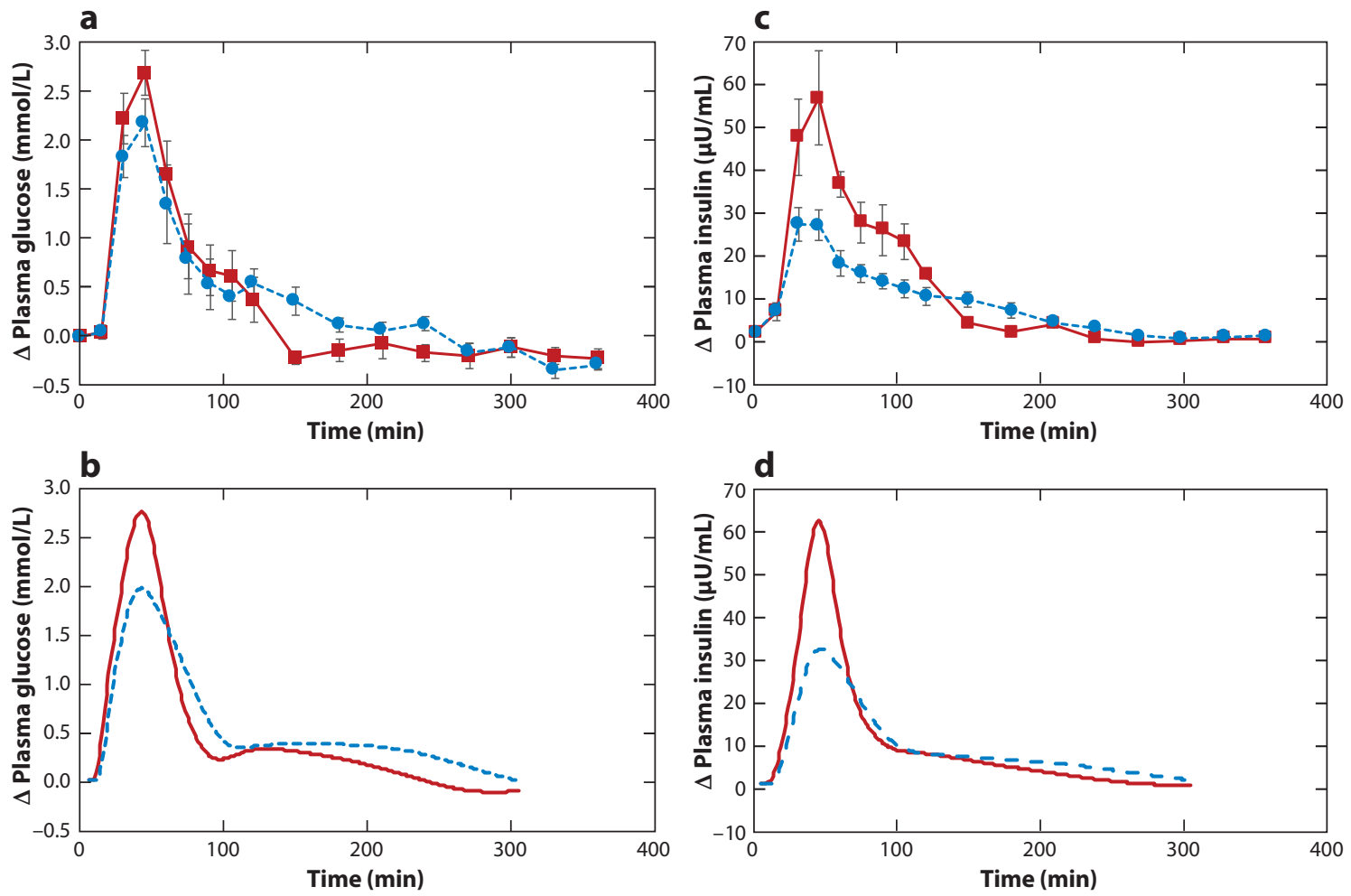

Figure 2

Example of digestion modeling by a program in development (van Aken 2020). (a,c) Experimental and (b,d) simulated blood glucose and insulin excursions following a bread meal (solid red line) and a pasta meal (dashed blue line). Experimental results have been adapted (with permission of Oxford University Press) from the study performed by Eelderink et al. (2012), who investigated a bread meal and a pasta meal, both consisting of $50 \mathrm{~g}$ of available carbohydrates, $9 \mathrm{~g}$ of fat, $6 \mathrm{~g}$ of protein, and $250 \mathrm{~mL}$ of tap water.

model, the differences between the two meals were predominantly caused by the differences in food structures. Compared to pasta (closed gel-like structure), the model assumes that bread (open sponge-like structure with more accessible starch) requires a longer time of oral processing and more saliva to obtain a swallowable bolus and is associated with faster starch hydrolysis and slower gastric-emptying kinetics. It is not the purpose of the present review to describe the model structure or to discuss the suitability of these modeling assumptions. Figure $\mathbf{2}$ is intended to provide only an illustration of what can be achieved with a physiologically based compartmental model of food digestion. It also provides a concrete illustration that the establishment of a comprehensive model of the functioning of the GI tract during human digestion can become a reality in the near future.

\subsection{Toward the Integration of Feedback Mechanisms}

The conditions in the alimentary system are highly influenced by the presence of food so as to adjust the physiological settings for optimal performance (van Aken 2010). Although physiological studies have delivered the detailed biological pathways for many of these regulatory mechanisms, mathematical modeling of these processes in relation to food digestion has remained rare. In the fed state, a large range of stimuli, including stomach and intestinal distension, luminal pressure, 
the presence of particles, acidity, osmotic value, and nutrient degradation products are detected by receptor cells all along the alimentary tract. These activate physiological responses through neural signals, sometimes directly, and sometimes indirectly through the nervus vagus, activated by secreting specific gut hormones, such as cholecystokinin (CCK), gastric inhibitory polypeptide (GIP), glucagon-like peptide-1 (GLP-1), peptide tyrosine (PYY), gastrin, motilin, and secretin (van Aken 2010).

Saliva secretion is stimulated by chewing, the parasympathic pathway, and various food stimuli (Ekström et al. 2012, Froehlich et al. 1987, Gavião \& Bilt 2004, Pandey et al. 2019). The secretion of gastric acid is stimulated by stomach distension, the presence of peptides in the stomach, and the buffering capacity of the food (Konturek et al. 1974) but is inhibited by a too low pH in the antrum (Wheeler 1974) and duodenum (Konturek \& Johnson 1971) due to the release of secretin and neural signals. Gastric acid secretion is also partly controlled by ghrelin, the hunger hormone, of which the blood levels increase due to the cephalic expectation of food (Arosio et al. 2004). Food material entering the small intestine is then detected by specific receptors that stimulate the secretion of pancreatic fluid and bile (Chandra \& Liddle 2015, Chey \& Chang 2001). These secretions are also mediated to a large extent by CCK (Chey \& Chang 2001, Liddle et al. 1985, Thimister et al. 1996), which is released by endocrine I-cells in the gut wall; neurons of the enteric nervous system and brain (Johnson 2014) in response to digestible proteins, peptides, and certain amino acids (Buffa et al. 1976, Johnson 2014); and fatty acids (Sidhu et al. 2000). Many other adjustments are related to the transition between fasted and fed states. The alimentary system even adjusts to returning dietary patterns by slowly modulating parameters such as receptor sensitivity and transporter presence in the gut (Baggio et al. 2004, Tong \& D'Alessio 2014).

Attempts to model some of these control mechanisms have been proposed in the literature by, for instance, Joseph et al. (2003) for the regulation of gastric acid secretion and Shiang \& Kandeel (2010) and Jelic et al. (2009) for the insulin regulatory system for blood plasma glucose and nonesterified fatty acids, respectively. As previously described, models of the physiological regulation of gastric emptying have also been proposed by Hunt \& Stubbs (1975) and Moxon et al. (2017). It is noteworthy that these models can remain rather mathematically simple despite the high diversity of the mechanisms involved. For instance, the in vivo regulation of gastric emptying is mediated by many receptors (Minami \& McCallum 1984), neural signals, and the release of intestinal hormones such as CCK, GLP-1, and ghrelin (Marathe et al. 2013, Minami \& McCallum 1984). Notwithstanding this biological complexity, Moxon et al. (2017) showed that the gastric-emptying patterns observed between low- and high-nutrient liquid meals could be accurately predicted by assuming an initial emptying rate followed by a maximal caloric flux into the duodenum. However, models of regulatory mechanisms remain scarce and can also be quite complex mathematically (e.g., see Joseph et al. 2003). Much more work is therefore needed to hierarchize the importance of control mechanisms and determine those that should be considered to reproduce the trends observed in vivo and whether and how they may be reproduced with simple modeling assumptions.

\subsection{Mathematical and Computational Challenges}

The preceding sections highlight promising first steps in establishing a mathematical modeling framework for food digestion and absorption built on compartmental approaches that have been successful in pharmacokinetics. These initial models typically focus on single nutrients, food structure, or microbiota, with a few more comprehensive models in animal feed science. However, to the authors' knowledge, no sustained attempt has been made to link these models together into a whole-body, all-nutrient model for human digestion and absorption that incorporates food structure information and microbiota; this is an indication of the mathematical challenges this task poses.

I6.I2 Le Feunteun et al. 
Table 1 gives insight into those challenges, with a list of 6 physiological compartments and 31 phenomena that the authors think should be modeled. Each of these phenomena adds its own set of equations and parameters to the modeling framework, leading to a multiparameter complex model. As noted at the end of Section 3.2, incorporating the modeling of postprandial metabolic responses to the absorption of just one nutrient can already lead to the explosion of physiological compartments and model parameters. For each nutrient and at various sections of the alimentary canal, there is a diverse range of phenomena to model, and one, therefore, sees that any model of digestion and absorption in humans would be complex.

This complexity generates several mathematical challenges, beginning with ensuring that appropriate equations are used to model the different phenomena. The task of translating information about digestion processes into appropriate equations where such equations do not yet exist requires excellent communication and collaboration between a variety of expert groups. Another mathematical challenge is the appropriate and physiologically sound determination of parameter values. Where experimental or otherwise reliable values are not readily available, more empirical techniques may need to be employed to set parameters, possibly leading to model inaccuracies. For example, in coupled differential equations with many parameter values to determine simultaneously, a common danger is finding a solution that is not the physiologically relevant optimum. Thus, the key challenge regarding parameters is how to determine physiologically correct values for those that cannot be determined easily, reliably, experimentally, or otherwise.

Moreover, a computational challenge related to the complexity of human food digestion and absorption model is its likely computational expensiveness and inefficiency. If existing software tools are linked to enact such a model, they would independently perform sometimes similar tasks without sharing efficiencies with other linked tools, and ultimately the efficiency of the whole process would be determined by the least efficient tool. Moreover, it would be difficult to manage different software tools working together in a properly interconnected manner. It is therefore likely that such a model should be developed from scratch to alleviate some of the computational complexity and efficiency issues. However, the time required to build and test such a model into a reasonable state for an application would likely be significant.

In this review, we have assumed that compartment models would be the framework for future human digestion and absorption models. Although the authors strongly believe this, there is the possibility that this may prove a major mathematical and computational challenge and that one may need to rely partially on other mathematical modeling paradigms, such as fluid dynamic models, which are not as easily linked to compartment models.

\subsection{Prospects on the Benefits of a More Complete Physiologically Based Food Digestion Model}

The building of a physiologically based model of the GI tract during digestion first requires gathering and carefully organizing the available knowledge. This crucial step represents a challenge (although surmountable) and an opportunity for researchers from various scientific fields (e.g., mathematics, nutrition, microbiology, human medical science, food and feed sciences, pharmacology) to share their knowledge and collaborate. This task would preferably be associated with the establishment of a dedicated multidisciplinary network and lead to a shared modeling platform that could serve as a basis for the incremental improvement of the model structure and underlying hypotheses. This would facilitate the identification of gaps, or gray boxes, of knowledge, hence possibly guiding the experimental research. This could even offer a common ground to bridge the gaps between food science and medical, pharmaceutical, and human microbiota sciences. Insight from food digestion studies can give a better and quantitative insight into the unabsorbed meal 
fraction reaching the colon (Beaumont et al. 2017), how food properties might modulate risk factors of metabolic diseases (e.g., diabetes type 2) and metabolic syndrome, and the way pharmaceuticals can be optimally delivered orally in conjunction with food intake (Koziolek et al. 2019). In return, the vast knowledge base of the medical field will deliver valuable insights into the development of healthier foods.

A more common hope with the establishment of a physiologically based model of digestion is that it could allow accurate in silico predictions about various aspects (Le Feunteun et al. 2020), probably starting with the effects of food/meal composition and structure on transit and disintegration kinetics, postprandial plasmatic concentrations in nutrients, and the arrival of unabsorbed nutrients and fibers available for the intestinal microbiota. Such a model could also be used to predict the effect of variations such as interindividual variability, time-of-day, and premeal effects and support the development of personalized nutrition, targeting different groups of the population. It could even become a central element in all models in which the processes taking place in the GI tract play a key role, as, for instance, for nutritional- (e.g., satiety), metabolic- (e.g., the fate of nutrients in the host), or colon microbiota (e.g., biodiversity)-related considerations. In the longterm, in silico predictions could even justify the need for in vivo investigations by fast evaluation of expected outcomes or alternatively offer a substitute to some animal and human studies (van Milgen \& Lescoat 2008).

The authors are convinced of the great possibility and benefits of developing a mathematical framework for performing in silico human food digestion and absorption experiments. Notwithstanding all the current challenges, the number of building blocks already existing, the youth of this research field, and the successes observed in the pharmaceutical area clearly support the idea that relevant scientific communities should gather and decide on a well-reasoned way to build (e.g., which modeling framework and assumptions?) and test (e.g., on which in vivo data?) such a model. It is difficult to know how accurate in silico predictions can become in the food digestion area, but our general impression is that this research field is mature enough to start building a human physiologically based food digestion model, if only to provide a practical means to bring research communities interested in the functioning of the GI closer.

\section{DISCLOSURE STATEMENT}

The authors are not aware of any affiliations, memberships, funding, or financial holdings that might be perceived as affecting the objectivity of this review.

\section{ACKNOWLEDGMENTS}

The authors would like to acknowledge INFOGEST COST Action FA1005 (2011-2015) and subsequently the INFOGEST network funded by INRAE for enabling the networking required to write this review.

\section{LITERATURE CITED}

Aarons L. 2005. Physiologically based pharmacokinetic modelling: a sound mechanistic basis is needed. $\mathrm{Br}$. . Clin. Pharmacol. 60(6):581-83

Arosio M, Ronchi CL, Beck-Peccoz P, Gebbia C, Giavoli C, et al. 2004. Effects of modified sham feeding on ghrelin levels in healthy human subjects. F. Clin. Endocrinol. Metab. 89(10):5101-4

Baggio LL, Kim J-G, Drucker DJ. 2004. Chronic exposure to GLP-1R agonists promotes homologous GLP-1 receptor desensitization in vitro but does not attenuate GLP-1R-dependent glucose homeostasis in vivo. Diabetes 53(Suppl. 3):S205-14

I6.I4 Le Feunteun et al. 
Baldini F, Heinken A, Heirendt L, Magnusdottir S, Fleming RMT, Thiele I. 2019. The Microbiome Modeling Toolbox: from microbial interactions to personalized microbial communities. Bioinforma. Oxf. Engl. 35(13):2332-34

Balsa-Canto E, Henriques D, Gábor A, Banga JR. 2016. AMIGO2, a toolbox for dynamic modeling, optimization and control in systems biology. Bioinformatics 32(21):3357-59

Bannink A, Dijkstra J, Koopmans S-J, Mroz Z. 2006. Physiology, regulation and multifunctional activity of the gut wall: a rationale for multicompartmental modelling. Nutr. Res. Rev. 19(2):227-53

Barros RM, Xavier Malcata F. 2004. A kinetic model for hydrolysis of whey proteins by cardosin A extracted from Cynara cardunculus. Food Chem. 88(3):351-59

Bastianelli D, Sauvant D, Rérat A. 1996. Mathematical modeling of digestion and nutrient absorption in pigs. 7. Anim. Sci. 74(8):1873-87

Beaumont M, Jaoui D, Douard V, Mat D, Koeth F, et al. 2017. Structure of protein emulsion in food impacts intestinal microbiota, caecal luminal content composition and distal intestine characteristics in rats. Mol. Nutr. Food Res. 61(10):1700078

Bohn T, Carriere F, Day L, Amelie D, Egger L, et al. 2018. Correlation between in vitro and in vivo data on food digestion. What can we predict with static in vitro digestion models? Crit. Rev. Food Sci. Nutr. 58(13):2239-61

Bornhorst GM, Gouseti O, Wickham MSJ, Bakalis S. 2016. Engineering digestion: multiscale processes of food digestion. 7. Food Sci. 81(3):R534-43

Brodkorb A, Egger L, Alminger M, Alvito P, Assunção R, et al. 2019. INFOGEST static in vitro simulation of gastrointestinal food digestion. Nat. Protoc. 14(4):991-1014

Buffa R, Solcia E, Go VLW. 1976. Immunohistochemical identification of the cholecystokinin cell in the intestinal mucosa. Gastroenterology 70(4):528-32

Chandra R, Liddle RA. 2015. Regulation of pancreatic secretion. Pancreapedia Exocrine Pancreas Knowl. Base. https://doi.org/10.3998/panc.2015.38

Chetty M, Johnson TN, Polak S, Salem F, Doki K, Rostami-Hodjegan A. 2018. Physiologically based pharmacokinetic modelling to guide drug delivery in older people. Adv. Drug Deliv. Rev. 135:85-96

Chey WY, Chang T. 2001. Neural hormonal regulation of exocrine pancreatic secretion. Pancreatology $1(4): 320-35$

Cremer J, Arnoldini M, Hwa T. 2017. Effect of water flow and chemical environment on microbiota growth and composition in the human colon. PNAS 114(25):6438-43

Csajka C, Drover D, Verotta D. 2005. The use of a sum of inverse Gaussian functions to describe the absorption profile of drugs exhibiting complex absorption. Pharm. Res. 22(8):1227-35

Dalla Man C, Camilleri M, Cobelli C. 2006. A system model of oral glucose absorption: validation on gold standard data. IEEE Trans. Biomed. Eng. 53(12):2472-78

Danhof M, de Lange ECM, Della Pasqua OE, Ploeger BA, Voskuyl RA. 2008. Mechanism-based pharmacokinetic-pharmacodynamic (PK-PD) modeling in translational drug research. Trends Pharmacol. Sci. 29(4):186-91

Drechsler KC, Bornhorst GM. 2018. Modeling the softening of carbohydrate-based foods during simulated gastric digestion. 7. Food Eng. 222:38-48

Drechsler KC, Ferrua MJ. 2016. Modelling the breakdown mechanics of solid foods during gastric digestion. Food Res. Int. 88:181-90

Dupont D, Alric M, Blanquet-Diot S, Bornhorst G, Cueva C, et al. 2019. Can dynamic in vitro digestion systems mimic the physiological reality? Crit. Rev. Food Sci. Nutr. 59(10):1546-62

Dupont D, Le Feunteun S, Marze S, Souchon I. 2018. Structuring food to control its disintegration in the gastrointestinal tract and optimize nutrient bioavailability. Innov. Food Sci. Emerg. Tecbnol. 46:83-90

Edwards CH, Warren FJ, Milligan PJ, Butterworth PJ, Ellis PR. 2014. A novel method for classifying starch digestion by modelling the amylolysis of plant foods using first-order enzyme kinetic principles. Food Funct. 5(11):2751-58

Eelderink C, Schepers M, Preston T, Vonk RJ, Oudhuis L, Priebe MG. 2012. Slowly and rapidly digestible starchy foods can elicit a similar glycemic response because of differential tissue glucose uptake in healthy men. Am. F. Clin. Nutr. 96(5):1017-24 
Egger L, Ménard O, Delgado-Andrade C, Alvito P, Assuncao R, et al. 2016. The harmonized INFOGEST in vitro digestion method: from knowledge to action. Food Res. Int. 88:217-25

Ekström J, Khosravani N, Castagnola M, Messana I. 2012. Saliva and the control of its secretion. In Dysphagia: Diagnosis and Treatment, ed. O Ekberg, pp. 19-47. Berlin: Springer

Elashoff JL, Reedy TJ, Meyer JH. 1982. Analysis of gastric emptying data. Gastroenterology 83:1306-12

Ferrua MJ, Singh RP. 2010. Modeling the fluid dynamics in a human stomach to gain insight of food digestion. 7. Food Sci. 75:R151-62

Ferrua MJ, Singh RP. 2015. Computational modelling of gastric digestion: current challenges and future directions. Curr. Opin. Food Sci. 4:116-23

Fouillet H, Juillet B, Gaudichon C, Mariotti F, Tome D, Bos C. 2009. Absorption kinetics are a key factor regulating postprandial protein metabolism in response to qualitative and quantitative variations in protein intake. Am. F. Physiol. Regul. Integr. Comp. Physiol. 297:R1691-705

Froehlich DA, Pangborn RM, Whitaker JR. 1987. The effect of oral stimulation on human parotid salivary flow rate and alpha-amylase secretion. Physiol. Behav. 41(3):209-17

Gavião MBD, der Bilt AV. 2004. Salivary secretion and chewing: stimulatory effects from artificial and natural foods. 7. Appl. Oral Sci. 12(2):159-63

Giang TM, Gaucel S, Brestaz P, Anton M, Meynier A, et al. 2016. Dynamic modeling of in vitro lipid digestion: individual fatty acid release and bioaccessibility kinetics. Food Chem. 194:1180-88

Guarner F, Malagelada J-R. 2003. Gut flora in health and disease. Lancet Lond. Engl. 361(9356):512-19

Haario H, Laine M, Mira A, Saksman E. 2006. DRAM: efficient adaptive MCMC. Stat. Comput. 16(4):339-54

Harrison SM, Cleary PW, Sinnott MD. 2018. Investigating mixing and emptying for aqueous liquid content from the stomach using a coupled biomechanical-SPH model. Food Funct. 9(6):3202-19

Harrison SM, Eyres G, Cleary PW, Sinnott MD, Delahunty C, Lundin L. 2014. Computational modeling of food oral breakdown using smoothed particle hydrodynamics. F. Texture Stud. 45(2):97-109

Heinken A, Sahoo S, Fleming RMT, Thiele I. 2013. Systems-level characterization of a host-microbe metabolic symbiosis in the mammalian gut. Gut Microbes 4(1):28-40

Heirendt L, Arreckx S, Pfau T, Mendoza SN, Richelle A, et al. 2019. Creation and analysis of biochemical constraint-based models using the COBRA Toolbox v.3.0. Nat. Protoc. 14(3):639-702

Hunt JN, Smith JL, Jiang CL. 1985. Effect of meal volume and energy density on the gastric emptying of carbohydrates. Gastroenterology 89(6):1326-30

Hunt JN, Stubbs DF. 1975. The volume and energy content of meals as determinants of gastric emptying. 7. Physiol. 245(1):209-25

INFOGEST In Silico Work. Group. 2019. A shared reference library on in silico models related to food digestion. INFOGEST. https://www.cost-infogest.eu/WORKING-GROUPS/WG6/A-sharedreference-library-on-in-silico-models-related-to-food-digestion

Iooss B, Janon A, Pujol G. 2019. R package "sensitivity.” https://cran.r-project.org/web/packages/ sensitivity/sensitivity.pdf

Iooss B, Lemaitre P. 2015. A review on global sensitivity analysis methods. In Uncertainty Management in Simulation-Optimization of Complex Systems, Vol. 59, ed. G Dellino, C Meloni, pp. 101-22. Boston, MA: Springer

Jelic K, Hallgreen CE, Colding-Jørgensen M. 2009. A model of NEFA dynamics with focus on the postprandial state. Ann. Biomed. Eng. 37(9):1897

Johnson LR. 2014. Gastrointestinal Physiology. Philadelphia: Elsevier Mosby. 8th ed.

Joseph IMP, Zavros Y, Merchant JL, Kirschner D. 2003. A model for integrative study of human gastric acid secretion. F. Appl. Physiol. 94(4):1602-18

Jullion A, Lambert P, Beck B, Vandenhende F. 2009. Pharmacokinetic parameters estimation using adaptive Bayesian P-splines models. Pharm. Stat. 8(2):98-112

Kettle H, Holtrop G, Louis P, Flint HJ. 2018. microPop: modelling microbial populations and communities in R. Methods Ecol. Evol. 9(2):399-409

Kettle H, Louis P, Holtrop G, Duncan SH, Flint HJ. 2015. Modelling the emergent dynamics and major metabolites of the human colonic microbiota. Environ. Microbiol. 17(5):1615-30

Kim WJ, Kim HU, Lee SY. 2017. Current state and applications of microbial genome-scale metabolic models. Curr. Opin. Syst. Biol. 2:10-18

I6.I6 Le Feunteun et al. 
Kondjoyan A, Daudin J-D, Santé-Lhoutellier V. 2015. Modelling of pepsin digestibility of myofibrillar proteins and of variations due to heating. Food Chem. 172:265-71

Konturek SJ, Biernat J, Oleksy J. 1974. Serum gastrin and gastric acid responses to meals at various $\mathrm{pH}$ levels in man. Gut 15(7):526-30

Konturek SJ, Johnson LR. 1971. Evidence for an enterogastric reflex for the inhibition of acid secretion. Gastroenterology 61(5):667-74

Kong F, Singh RP. 2009. Modes of disintegration of solid foods in simulated gastric environment. Food Biophys. 4(3):180-90

Korpela K. 2018. Diet, microbiota, and metabolic health: trade-off between saccharolytic and proteolytic fermentation. Annu. Rev. Food Sci. Technol. 9:65-84

Koziolek M, Alcaro S, Augustijns P, Basit AW, Grimm M, et al. 2019. The mechanisms of pharmacokinetic food-drug interactions: a perspective from the UNGAP group. Eur. F. Pharm. Sci. 134:31-59

Labarthe S, Polizzi B, Phan T, Goudon T, Ribot M, Laroche B. 2019. A mathematical model to investigate the key drivers of the biogeography of the colon microbiota. F. Theor. Biol. 462:552-81

Lavielle M. 2014. Mixed Effects Models for the Population Approach: Models, Tasks, Methods and Tools. Boca Raton, FL: CRC Press

Le Feunteun S, Barbe F, Remond D, Menard O, Le Gouar Y, et al. 2014. Impact of the dairy matrix structure on milk protein digestion kinetics: mechanistic modelling based on mini-pig in vivo data. Food Bioprocess Technol. 7:1099-113

Le Feunteun S, Mackie AR, Dupont D. 2020. In silico trials of food digestion and absorption: How far are we? Curr. Opin. Food Sci. 31:121-25

Li H, Dhital S, Gidley MJ, Gilbert RG. 2019. A more general approach to fitting digestion kinetics of starch in food. Carbohydr. Polym. 225:115244

Li J, Jia H, Cai X, Zhong H, Feng Q, et al. 2014. An integrated catalog of reference genes in the human gut microbiome. Nat. Biotecbnol. 32(8):834-41

Li M, Zhao P, Pan Y, Wagner C. 2018. Predictive performance of physiologically based pharmacokinetic models for the effect of food on oral drug absorption: current status. CPT Pharmacomet. Syst. Pharmacol. $7(2): 82-89$

Liddle RA, Goldfine ID, Rosen MS, Taplitz RA, Williams JA. 1985. Cholecystokinin bioactivity in human plasma. Molecular forms, responses to feeding, and relationship to gallbladder contraction. 7 . Clin. Invest. 75(4):1144-52

Luo Q, Zhan W, Boom RM, Janssen AEM. 2018. Interactions between acid and proteins under in vitro gastric condition: a theoretical and experimental quantification. Food Funct. 9(10):5283-89

Manca D, ed. 2018. Quantitative Systems Pharmacology: Models and Model-Based Systems with Applications. Amsterdam: Elsevier. 1st ed.

Marathe CS, Rayner CK, Jones KL, Horowitz M. 2013. Relationships between gastric emptying, postprandial glycemia, and incretin hormones. Diabetes Care 36(5):1396-405

Mc Auley MT, Mooney KM. 2015. Computationally modeling lipid metabolism and aging: a mini-review. Comput. Struct. Biotechnol. 7. 13:38-46

Mennah-Govela YA, Keppler S, Januzzi-Guerreiro F, Follador-Lemos C, Vilpont K, Bornhorst GM. 2020. Acid and moisture uptake into red beets during in vitro gastric digestion as influenced by gastric $\mathrm{pH}$. Food Biophys. 15:261-72

Mennah-Govela YA, Singh RP, Bornhorst GM. 2019. Buffering capacity of protein-based model food systems in the context of gastric digestion. Food Funct. 10(9):6074-87

Meraz M, Alvarez-Ramirez J, Vernon-Carter EJ, Reyes I, Hernandez-Jaimes C, Martinez-Martinez F. 2019. A two competing substrates Michaelis-Menten kinetics scheme for the analysis of in vitro starch digestograms. Starch 72(9-10):1900170

Minami H, McCallum RW. 1984. The physiology and pathophysiology of gastric emptying in humans. Gastroenterology 86:1592-610

Minekus M, Alminger M, Alvito P, Ballance S, Bohn T, et al. 2014. A standardised static in vitro digestion method suitable for food: an international consensus. Food Funct. 5:1113-24

Morris MD. 1991. Factorial sampling plans for preliminary computational experiments. Tecbnometrics 33(2):161-74 
Motelica-Wagenaar AM, Nauta A, van den Heuvel EGHM, Kleerebezem R. 2014. Flux analysis of the human proximal colon using anaerobic digestion model 1. Anaerobe 28:137-48

Moxon TE, Gouseti O, Bakalis S. 2016. In silico modelling of mass transfer \& absorption in the human gut. 7. Food Eng. 176:110-20

Moxon TE, Nimmegeers P, Telen D, Fryer PJ, Van Impe J, Bakalis S. 2017. Effect of chyme viscosity and nutrient feedback mechanism on gastric emptying. Chem. Eng. Sci. 171:318-30

Mulet-Cabero A-I, Egger L, Portmann R, Ménard O, Marze S, et al. 2020. A standardised semi-dynamic in vitro digestion method suitable for food: an international consensus. Food Funct. 11(2):1702-20

Muñoz-Tamayo R, Giger-Reverdin S, Sauvant D. 2016. Mechanistic modelling of in vitro fermentation and methane production by rumen microbiota. Anim. Feed Sci. Technol. 220:1-21

Muñoz-Tamayo R, Laroche B, Walter E, Dore J, Leclerc M. 2010. Mathematical modelling of carbohydrate degradation by human colonic microbiota. F. Theor. Biol. 266:189-201

Muttakin S, Moxon TE, Gouseti O. 2019. In vivo, in vitro, and in silico studies of the GI tract. In Interdisciplinary Approaches to Food Digestion, ed. O Gouseti, GM Bornhorst, S Bakalis, A Mackie, pp. 29-67. Cham., Switz.: Springer

Oberli M, Douard V, Beaumont M, Jaoui D, Devime F, et al. 2018. Lipo-protein emulsion structure in the diet affects protein digestion kinetics, intestinal mucosa parameters and microbiota composition. Mol. Nutr. Food Res. 62(2):1700570

Orth JD, Thiele I, Palsson BØ. 2010. What is flux balance analysis? Nat. Biotechnol. 28(3):245-48

Pandey M, Reddy V, Wanjari P. 2019. Comparative evaluation of citric acid and TENS as means for salivary stimulation in adults: an in vivo study. J. Indian Acad. Oral Med. Radiol. 31(1):36-39

Pentafragka C, Symillides M, McAllister M, Dressman J, Vertzoni M, Reppas C. 2019. The impact of food intake on the luminal environment and performance of oral drug products with a view to in vitro and in silico simulations: a PEARRL review. F. Pharm. Pharmacol. 71(4):557-80

Raguideau S, Plancade S, Pons N, Leclerc M, Laroche B. 2016. Inferring aggregated functional traits from metagenomic data using constrained non-negative matrix factorization: application to fiber degradation in the human gut microbiota. PLOS Comput. Biol. 12(12):e1005252

Raue A, Steiert B, Schelker M, Kreutz C, Maiwald T, et al. 2015. Data2Dynamics: a modeling environment tailored to parameter estimation in dynamical systems. Bioinformatics 31(21):3558-60

Rivest J, Bernier JF, Pomar C. 2000. A dynamic model of protein digestion in the small intestine of pigs. 7. Anim. Sci. 78:328-40

Rozendaal YJ, Maas AH, van Pul C, Cottaar EJ, Haak HR, et al. 2018. Model-based analysis of postprandial glycemic response dynamics for different types of food. Clin. Nutr. Exp. 19:32-45

Saltelli A, Ratto M, Andres T, Campolongo F, Cariboni J, et al. 2008. Global Sensitivity Analysis. The Primer. Chichester, UK: Wiley \& Sons

Saturnino GB, Thielscher A, Madsen KH, Knösche TR, Weise K. 2019. A principled approach to conductivity uncertainty analysis in electric field calculations. NeuroImage 188:821-34

Shiang K-D, Kandeel F. 2010. A computational model of the human glucose-insulin regulatory system. 7. Biomed. Res. 24(5):347-64

Sicard J, Mirade P-S, Portanguen S, Clerjon S, Kondjoyan A. 2018. Simulation of the gastric digestion of proteins of meat bolus using a reaction-diffusion model. Food Funct. 9(12):6455-69

Sidhu SS, Thompson DG, Warhurst G, Case RM, Benson RSP. 2000. Fatty acid-induced cholecystokinin secretion and changes in intracellular $\mathrm{Ca}^{2+}$ in two enteroendocrine cell lines, STC-1 and GLUTag. 7. Physiol. 528(Pt. 1):165-76

Siegel JA, Urbain JL, Adler LP, Charkes ND, Maurer AH, et al. 1988. Biphasic nature of gastric emptying. Gut 29(1):85-89

Strathe AB, Danfær A, Chwalibog A. 2008. A dynamic model of digestion and absorption in pigs. Anim. Feed Sci. Technol. 143:328-71

Sudret B. 2008. Global sensitivity analysis using polynomial chaos expansions. Reliab. Eng. Syst. Saf. 93(7):96479

Taghipoor M, Barles G, Georgelin C, Licois JR, Lescoat P. 2014. Digestion modeling in the small intestine: impact of dietary fiber. Math. Biosci. 258:101-12 
Taghipoor M, Lescoat P, Licois JR, Georgelin C, Barles G. 2012. Mathematical modeling of transport and degradation of feedstuffs in the small intestine. F. Theor. Biol. 294:114-21

Thiele I, Palsson BØ. 2010. A protocol for generating a high-quality genome-scale metabolic reconstruction. Nat. Protoc. 5(1):93-121

Thimister P, Hopman W, Sloots C, Rosenbusch G, Willems H, et al. 1996. Role of intraduodenal proteases in plasma cholecystokinin and pancreaticobiliary responses to protein and amino acids. Gastroenterology 110(2):567-75

Tong J, D'Alessio D. 2014. Give the receptor a brake: slowing gastric emptying by GLP-1. Diabetes 63(2):407-9 Usry JL, Turner LW, Stahly TS, Bridges TC, Gates RS. 1991. GI tract simulation-model of the growing pig. Trans. ASAE 34:1879-90

van Aken GA. 2010. Relating food emulsion structure and composition to the way it is processed in the gastrointestinal tract and physiological responses: What are the opportunities? Food Biophys. 5(4):258-83

van Aken GA. 2020. In silico digestive physiology modeling. Insight Food Inside. http://www. insightfoodinside.com/Food-interacting-with-the-body/In-silico-digestion-modelling/

van Bentum R, Nelson MI. 2011. Modelling the passage of food through an animal stomach: a chemical reactor engineering approach. Chem. Eng. F. 166:315-23

van der Graaf PH, Benson N. 2011. Systems pharmacology: bridging systems biology and pharmacokineticspharmacodynamics (PKPD) in drug discovery and development. Pharm. Res. 28(7):1460-64

van der Sman RGM, Houlder S, Cornet S, Janssen A. 2020. Physical chemistry of gastric digestion of proteins gels. Curr. Res. Food Sci. 2:45-60

van Milgen J, Lescoat P. 2008. Modelling digestion and metabolism in the pig: an alternative to animal experimentation? Bull. Acad. Vet. Fr. 161:435-40

Wheeler MH. 1974. Inhibition of gastric secretion by the pyloric antrum. Gut 15(5):420-32

Xiao L, Estellé J, Kiilerich P, Ramayo-Caldas Y, Xia Z, et al. 2016. A reference gene catalogue of the pig gut microbiome. Nat. Microbiol. 1(12):16161

Xiao L, Feng Q, Liang S, Sonne SB, Xia Z, et al. 2015. A catalog of the mouse gut metagenome. Nat. Biotechnol. 33(10):1103-8

Yu LX. 1999. An integrated model for determining causes of poor oral drug absorption. Pharm. Res. 16:188387

Zhuang X, Lu C. 2016. PBPK modeling and simulation in drug research and development. Acta Pharm. Sin. B. 6(5):430-40 